\title{
A very young component in the pre-eminent starburst region of the Small Magellanic Cloud
}

\author{
M. Heydari-Malayeri and R. Selier
}

\begin{abstract}
Laboratoire d'Etudes du Rayonnement et de la Matière en Astrophysique (LERMA), Observatoire de Paris, CNRS, 61 Avenue de l'Observatoire, 75014 Paris, France

e-mail: m.heydari@obspm.fr
\end{abstract}

Received 10 February 2010 / Accepted 14 April 2010

\section{ABSTRACT}

\begin{abstract}
Context. Despite extensive research on various components of the N66/NGC 346 complex, few studies have so far focused on N66A, which is a special object in the whole complex and therefore deserves scrutiny. The study of this compact H II region and its fellow objects seems important in the framework of massive star formation in the Magellanic Clouds.

Aims. We present a study of the compact H II region N66A in the SMC pre-eminent starburst region N66/NGC 346.

Methods. This analysis is based mainly on our optical ESO NTT observations, both imaging and spectroscopy, coupled with archive HST ACS data and Spitzer infrared images (IRAC 3.6, 4.5, 5.8, and $8.0 \mu \mathrm{m}$ ).

Results. We derive a number of physical characteristics of the compact H II region N66A. For the first time using spectroscopy, we present the spectral classification of the main exciting star of N66A. Its spectral features indicate that it is a main-sequence massive star of type O8. We compare this result with that based on the stellar Lyman continuum flux estimated from the ionized gas $\mathrm{H} \beta$ flux. The compact H II region belongs to a rare class of $\mathrm{H}$ II regions in the Magellanic Clouds, called high-excitation blobs (HEBs). We propose that N66A probably represents a very young massive star formation event in the N66 complex that has a range of ages.
\end{abstract}

Key words. H II regions - stars: early-type - stars: formation - stars: fundamental parameters - ISM: individual objects: N66A Magellanic Clouds

\section{Introduction}

LHA 115-N66, or in short N66 (Henize 1956), is the largest and the most luminous H II region in the Small Magellanic Cloud (SMC). It is also known as DEM S 103 (Davies et al. 1976) and NGC 346, the latter referring to the bright $\mathrm{OB}$ association located at its center. We refer the reader to the magnificent images of the region taken with the Advanced Camera for Surveys (ACS) onboard the Hubble Space Telescope and the $J H K$ composite obtained with ISAAC on the ESO VLT telescope (Nota et al. 2006; Gouliermis et al. 2010). N66 is considered to be the scaled-down counterpart of the Large Magellanic Cloud starburst 30 Doradus. It indeed hosts the largest sample of young, massive stars in the whole SMC with 33 O-type stars among which 11 are of type O6.5 or earlier (Massey et al. 1989; Walborn et al. 2000; Evans et al. 2006). It contains at least one W-R star in the massive binary or maybe triple system HD 5980. An age of $~ 3 \mathrm{Myr}$ has been estimated for NGC 346 from evolutionary models in the H-R diagram (Bouret et al. 2003). HD 5980 lies behind a SNR (B0057-724, Reid et al. 2006; Nazé et al. 2002; Danforth et al. 2003; Nazé et al. 2004), which has no known optical counterpart. Compared to the Orion Nebula, N66 has an $\mathrm{H} \alpha$ luminosity almost 60 times higher (Kennicutt 1984). This radiant flux is also reminiscent of those of giant $\mathrm{H}$ II regions in distant metal-poor galaxies, such as regions A1 and A2 in IC 4662 lying 2.44 Mpc away (Heydari-Malayeri et al. 1990; Crowther \& Bibby 2009, and references therein). Therefore, N66 offers a valuable template for studying these kinds of distant galaxies with high resolution.

Apart from its recently formed massive star population mentioned above, NGC 346 also has a large population of low-mass, pre-main-sequence stars (Gouliermis et al. 2006; Nota et al. 2006; Sabbi et al. 2007; Hennekemper et al. 2008) covering a mass range down to the subsolar regime. The PMS population is found to be mainly concentrated in a number of subclusters away from the massive star association (Sabbi et al. 2007; Schmeja et al. 2000). The typical ages of the PMS population derived from models appear to suggest that low-mass star formation events occurred at two different epochs about 4 and $10 \mathrm{Myr}$ ago (Hennekemper et al. 2008).

Being such an extraordinary object, the N66 complex has been extensively studied from various viewpoints using almost the whole electromagnetic range, from radio wavelengths to $\mathrm{X}$-rays. More specifically, the studies have used the radio continuum (Ye et al. 1991; Reid et al. 2006) and the $21 \mathrm{~cm}$ atomic line (Staveley-Smith et al. 1997; Stanimirović et al. 1999), the CO line with the SEST telescope (Rubio et al. 1996, 2000), the infrared with both the Infrared Space Observatory (Contursi et al. 2000) and the Spitzer Space Telescope (Bolatto et al. 2007; Simon et al. 2007), optical spectroscopy (Massey et al. 1989; Walborn et al. 2000; Evans et al. 2006), ultraviolet and optical spectra (Walborn et al. 2000), with data from the HST spectrograph STIS, AAT, and ESO $3.6 \mathrm{~m}$ telescope, imaging with ACS and WFC on board HST (Nota et al. 2006), H $\alpha$ (e.g., Kennicutt 1984; Le Coarer et al. 1993; Smith et al. 2000), in the UV with the International Ultraviolet Explorer (de Boer \& Savage 1980) and the Far-Ultraviolet Spectroscopic Explorer (Danforth et al. 2003), and in the X-rays with XMM-Newton and Chandra (Nazé et al. 2002, 2004).

The present study is concerned with massive star formation in the N66 complex. Clustered mainly in NGC 346, as 
mentioned above, massive stars dominate the central part of the whole $\mathrm{H}$ II region with their strong UV radiation field. Twentytwo of the above-mentioned $33 \mathrm{O}$ stars are contained in the central cluster. The hottest star, W3, is reclassified as O2 III(f*) (Walborn et al. 2002a,b). The most massive star, W1, of the central cluster, classified O4 III(n)(f) (Walborn \& Blades 1986), has multiple components and the mass of the brightest component is at most $85 M_{\odot}$ (Heydari-Malayeri \& Hutsemékers 1991). The cluster has disrupted the bulk of the natal molecular cloud, and therefore not much $\mathrm{CO}$ emission is detected towards N66, except for two positions which are mapped in the (1-0) and (2-1) transitions (Rubio et al. 1996, 2000).

One of these CO peaks is associated with a remarkable feature of the whole landscape, a compact H II region, called N66A, according to Henize (1956). The H II region apparently lies at the south-eastern end of an absorption lane that runs over some 60 pc from north-west to south-east below the NGC 346 cluster. This paper is mainly devoted to this compact H II region. Despite extensive research on various components of the N66/NGC 346 complex, few studies have so far dealt with this $\mathrm{H}$ II region. We attempt to demonstrate that this region represents the youngest episode of massive star formation in N66.

A word of caution seems necessary about the name of this object. From their observations of $\mathrm{H}_{2}$ emission line $(2.14 \mu \mathrm{m})$ and the ISOCAM LW2 band (centered at $6.75 \mu \mathrm{m}$ ), Contursi et al. (2000) and Rubio et al. (2000) detected several embedded sources towards N66, which they alphabetically designated from "A" to "I". The IR source A should not be confused with the Henize N66A H II component, which corresponds to the IR source "H". In a similar way, NGC 346 corresponds to "C".

The paper is organized as follows. Section 2 presents the observations, data reduction, and the archive data used (HST ACS data, Spitzer data). Section 3 describes our results (morphology, physical parameters, spectral classification). Section 4 presents our discussion, and finally our conclusions are summarized in Sect. 5.

\section{Observations and data reduction}

\subsection{NTT Imaging}

N66 was observed on 28 September 2002 using the ESO New Technology Telescope (NTT) equipped with the active optics and the SUperb Seeing Imager (SuSI2). The detector consisted of two CCD chips, identified as ESO \#45 and \#46. The two resulting frames were automatically combined in a single FITS file, whereas the space between the two chips was "filled" with some overscan columns so that the respective geometry of the two chips was approximately preserved. The gap between the chips corresponds to $\sim 100$ true CCD pixels, or $\sim 8^{\prime \prime}$. The file format was $4288 \times 4096$ pixels, and the measured pixel size $0{ }^{\prime} .085$ on the sky. Each chip of the mosaic covered a field of $5.5 \times 2$ '.7. We refer to the ESO manual SuSI2 for more technical information (LSO-MAN-ESO-40100-0002/1.9).

Nebular imaging was carried out using the narrow-band filters centered on the emission lines $\mathrm{H} \alpha$ (ESO \#884), $\mathrm{H} \beta$ (\#881), and [O III] (\#882) with three basic exposures of $300 \mathrm{~s}$. The image quality was quite good during the night, being represented by a seeing of $0 . ' 5-0$. $^{\prime} 8$ (Fig. 1). We constructed the line ratio maps $\mathrm{H} \alpha / \mathrm{H} \beta$ and $[\mathrm{O} \mathrm{III}] / \mathrm{H} \beta$ from nebular imaging. We also took two exposures using filters ESO \#811 (B), \#812 (V), and \#813 $(R)$ with unit exposure times of $15 \mathrm{~s}$ for $B$ and $V$ and $10 \mathrm{~s}$ for $R$, respectively. The exposures for each filter were repeated two times using ditherings of $5^{\prime \prime}-10^{\prime \prime}$ for bad pixel rejection.

\subsection{NTT spectroscopy}

The EMMI spectrograph attached to the ESO NTT telescope was used on 29 September 2002 to obtain several long-slit spectra of the stars. The grating was \# 12 centered on $4350 \AA$ (BLMRD mode) and the detector was a Tektronix CCD TK1034 with $1024^{2}$ pixels of size $24 \mu \mathrm{m}$. The covered wavelength range was 3810-4740 $\AA$ and the dispersion $38 \AA \mathrm{mm}^{-1}$, giving $F W H M$ resolutions of $2.70 \pm 0.10$ pixels or $2.48 \pm 0.13 \AA$ for a $11^{\prime \prime} 0$ slit. At each position, we took three $10 \mathrm{~min}$ exposure. The instrument response was derived from observations of the calibration stars LTT 7379, LTT 6248, and LTT 7987. The seeing conditions varied between 0.9 and $11^{\prime \prime} 2$. The identifications of the stars along the slits were based on monitor sketches drawn during the observations.

Furthermore, EMMI was used on 26 and 27 September 2002 to obtain nebular spectra with gratings \# 8 (4550-6650 $\AA$ ) and \# 13 (4200-8000) in the REMD mode and with grating \#4 (3650-5350 $\AA$ ) in the BLMD mode. In the REMD mode, the detector was CCD \# 63, MIT/LL, $2048 \times 4096$ pixels of $15^{2} \mu \mathrm{m}^{2}$ each. Spectra were obtained with the slit set in east-west and north-south orientations using a basic exposure time of $300 \mathrm{~s}$ repeated several times. The seeing conditions varied between $0 .{ }^{\prime} 6$ and 0.8 . Reduction and extraction of spectra were performed using the IRAF software package. Fluxes were derived from the extracted spectra with the IRAF task SPLOT. The line fluxes were measured by fitting Gaussian profiles to the lines as well as by simple pixel integration in some cases. The nebular line intensities were corrected for interstellar reddening using the formulae given by Howarth (1983) for the LMC extinction.

\subsection{Archive data}

\subsubsection{HST ACS data}

We used archive imaging data of NGC 346 (GO 10248, P.I. A. Nota). These observations were obtained with ACS (Advanced Camera for Surveys) onboard HST. The images were taken with the Wide Field Camera (WFC) using broad- and narrow-band filters $(F 555 W, F 814 W, F 656 N)$ in 2004 July. They were used to examine the morphology of the compact H II region N66A and in particular resolve its exciting stars. In addition, we produced a composite image of N66A (Figs. 2, 3) and also used the photometry derived from these observations (Gouliermis 2006). We also used the archive observations obtained in July 2006 using ACS with the High Resolution Channel (HRC) and the ultraviolet filters $F 220 W$ and $F 330 W$ (GO 10542, P.I. A. Nota).

\subsubsection{Spitzer data}

The Spitzer archive data used in this paper come from the $S^{3} \mathrm{MC}$ project. This is a project to map the star-forming body of the SMC with Spitzer in all seven Infrared Array Camera (IRAC) and Multiband Imaging Photometer for Spitzer (MIPS) bands. The MIPS data were obtained in 2004 November and the IRAC data in 2005 May. We used the IRAC data to build a composite image of N66 and also obtain the photometry of N66A. The typical PSF of the IRAC images in the 3.6, 4.5, 5.8, and $8.0 \mu \mathrm{m}$ bands is 1 '.66 to 1 '.98 and that of MIPS at $24 \mu \mathrm{m}$ is $6^{\prime \prime}$ (Bolatto et al. 2007). The derived photometry for N66A in the 3.6, 4.5, 5.8, and $8.0 \mu \mathrm{m}$ bands are $12.22,11.82,10.25$, and $8.66 \mathrm{mag}$, respectively, using an integration aperture of 5 pixels, or 6 " in radius. Measurements with either slightly larger or smaller apertures do 


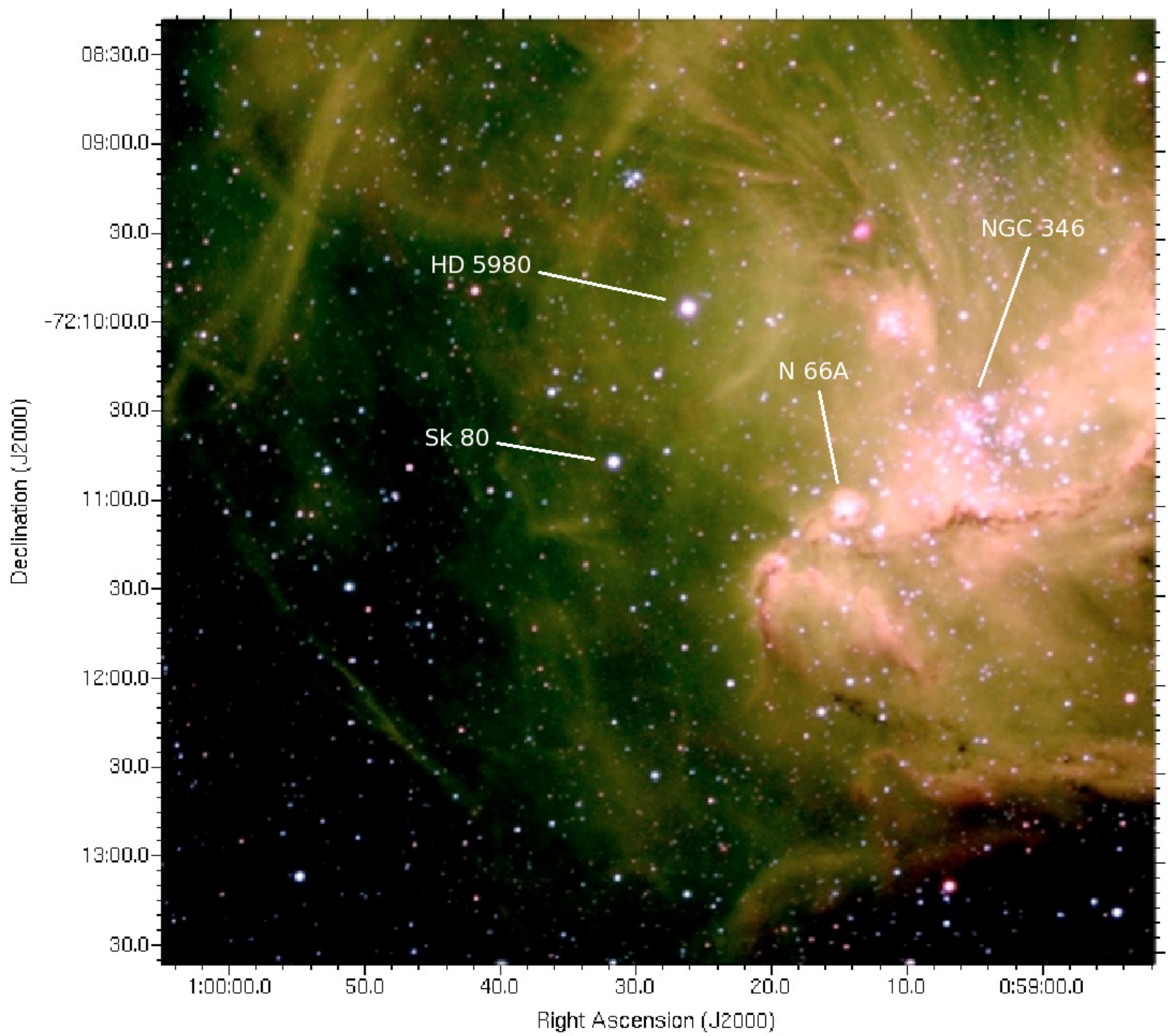

Fig. 1. A composite three-color image of the SMC H II region N66. The star cluster above the curling absorption lane is the OB association NGC 346. N66A is the brightest compact H II region lying at the eastern end of the dark lane. Note the wind-driven bubble centered on the brightest star HD 5980. The other bright star lying towards the field center is Sk 80. The image, taken with the ESO NTT/SuSI2, results from the coaddition of narrow-band filters $\mathrm{H} \alpha$ (red), [O III] (green), and $\mathrm{H} \beta$ (blue). The field size is $336^{\prime \prime} \times 350^{\prime \prime}$ corresponding to $100 \times 103 \mathrm{pc}$. North is up and east to the left.

not affect the color results. Moreover, we tried to detect substructures in the Spitzer images of N66A (see Sect. 4).

\section{Results}

\subsection{Morphology}

Figure 1 displays a composite image of the N66 region taken with the NTT telescope (see Sect. 2.1). The field is $\sim 5^{\prime} \times 5^{\prime}$ corresponding to $\sim 90 \mathrm{pc}^{2}$ for a distance of about $60 \mathrm{kpc}$, or $m-M=18.94$ mag (Laney \& Stobie 1994). Although the resolution is less than that of the HST ACS image (Sect. 2.3.1), almost all the features of the H II complex are visible. The NGC 346 cluster appears to be at the center of an H II bowl, the southern border of which is delineated by a compressed ionized gas front and an absorption lane running over some $60 \mathrm{pc}$. In particular, N66A stands out as the most compact H II nebula of the whole region, with coordinates (J2000.0) $\alpha=00: 59: 14.8$, $\delta=-72: 11: 01$. The compact H II region is apparently associated with the compressed gas front and the absorption lane.

The field of view of the NTT image is larger than that of HST ACS. It also displays a turbulent environment in the eastern side of N66 with many indications of shocked gas. In particular, the wind-driven bubble centered on HD 5980 is quite impressive. A narrow ridge can also be discerned towards the southern outer boundary of the complex. This feature is also affected by stellar shock winds, as indicated by its remarkable [S II] emission (Reid et al. 2006). Observations with the Australia Telescope Compact Array (ATCA) and Parkes Observatory at the Australia Telescope National Facility (ATNF) also detect an H I cloud 

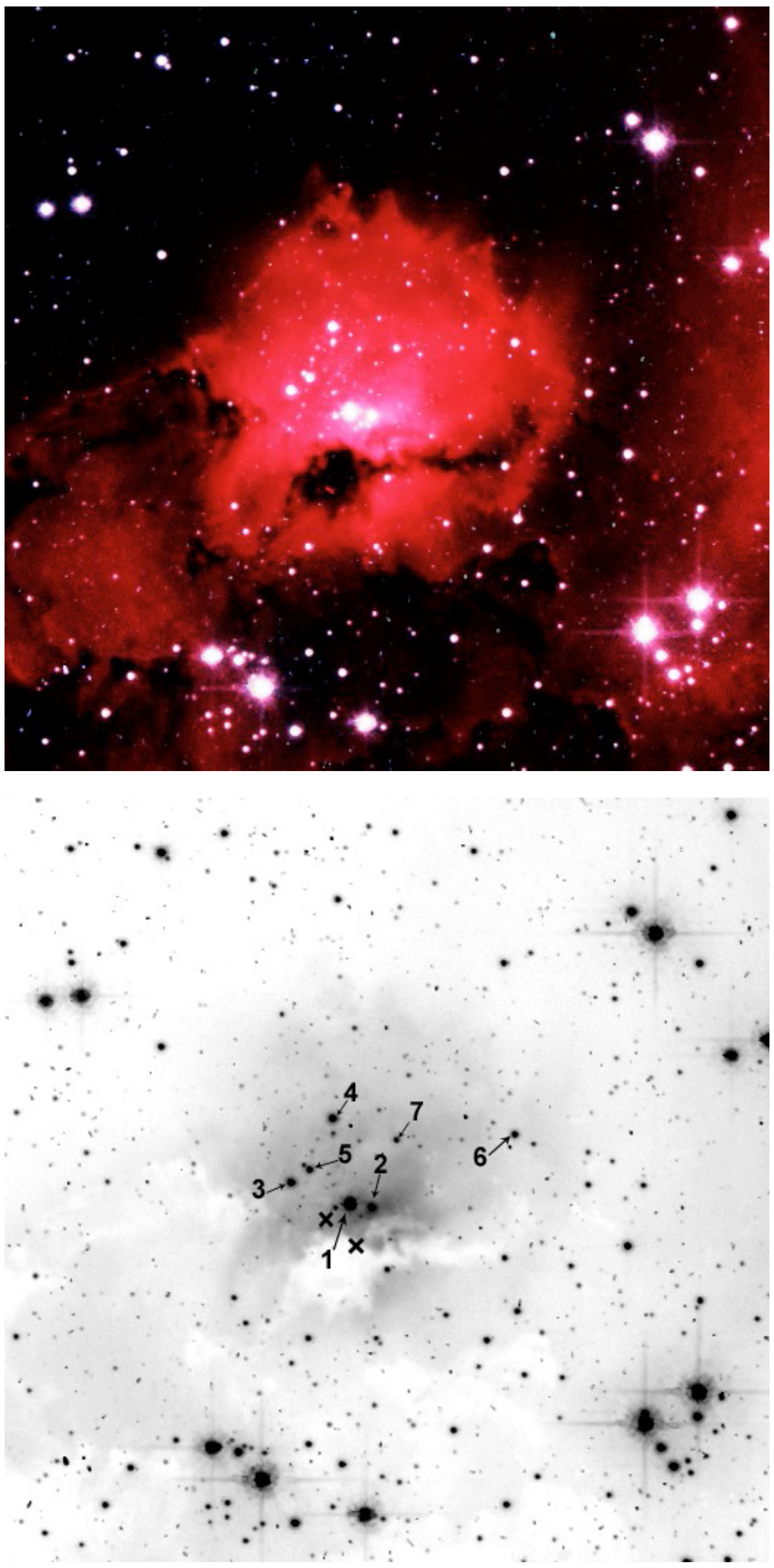

Fig. 2. A composite three-color image of SMC N66A created using the HST ACS images in $\mathrm{H} \alpha$ (red), filter $I, F 814 W$ (green), and filter $V$, $F 555 W$ (blue). Field size $521 \times 521$ pixels, or $26^{\prime \prime} \times 26^{\prime \prime}(\sim 7.5 \times 7.5 \mathrm{pc})$. North is up and east to the left.
Fig. 3. The N66A image of SMC N66A extracted from the HST ACS image obtained through filter $V(F 555 W)$. The main stars associated with the H II region are marked by numbers; see also Table 2. The crosses represent the two YSO positions as suggested by Spitzer observations (Simon et al. 2007). Field size and orientation same as in Fig. 2. 
Table 1. Some physical parameters of N66A.

\begin{tabular}{cccccccc}
\hline \hline $\begin{array}{c}\theta \\
(\prime)\end{array}$ & $\begin{array}{c}r \\
(\mathrm{pc})\end{array}$ & $\begin{array}{c}I(\mathrm{H} \beta)^{\dagger} \\
\mathrm{erg} \mathrm{s}^{-1} \mathrm{~cm}^{-2} \\
\times 10^{-12}\end{array}$ & $\begin{array}{c}T e \\
(\mathrm{~K})\end{array}$ & $\begin{array}{c}\mathrm{Ne} \\
\mathrm{cm}^{-3}\end{array}$ & $\begin{array}{c}\left\langle n_{\mathrm{e}}\right\rangle \\
\mathrm{cm}^{-3}\end{array}$ & $\begin{array}{c}M_{\text {gas }} \\
\left(M_{\odot}\right)\end{array}$ & $\begin{array}{c}N_{L} \\
\mathrm{ph} \mathrm{s}^{-1} \\
\times 10^{48}\end{array}$ \\
\hline 5 & 1.5 & 3.6 & 12770 & 220 & 200 & 90 & 3.4 \\
\hline
\end{tabular}

Notes. $\dagger$ Using a reddening coefficient $c(\mathrm{H} \beta)=0.26$.

Table 2. Positions and photometry of stars associated with N66A.

\begin{tabular}{ccccccccr}
\hline \hline Star ID & $\alpha(2000)$ & $\delta(\mathrm{J} 2000)$ & $F 220 W$ & $F 330 W$ & $V$ & $I$ & $(F 220 W-V)$ & Other designations $^{\ddagger}$ \\
\hline N66A-1 & $00: 59: 14.93$ & $-72: 11: 01.90$ & 13.89 & 14.63 & 15.97 & 16.15 & -2.08 & G 67; MPG 646 \\
N66A-2 & $00: 59: 14.77$ & $-72: 11: 01.97$ & 15.64 & 16.34 & 17.33 & 17.41 & -1.69 & G 271; MPG 641 \\
N66A-3 & $00: 59: 15.38$ & $-72: 11: 01.25$ & 16.26 & 16.86 & 17.77 & 17.94 & -1.51 & G 427; MPG 654 \\
N66A-4 & $00: 59: 15.10$ & $-72: 10: 59.02$ & 16.68 & 17.15 & 17.82 & 17.87 & -1.14 & G 414; MPG 648 \\
N66A-5 & $00: 59: 15.25$ & $-72: 11: 00.78$ & 18.56 & 18.59 & 18.74 & 18.45 & -0.18 & G 844; MPG 648 \\
N66A-6 & $00: 59: 13.74$ & $-72: 10: 59.23$ & 18.22 & 18.54 & 18.98 & 19.05 & -0.76 & G 1480; MPG 629 \\
N66A-7 & $00: 59: 14.61$ & $-72: 10: 59.63$ & 19.14 & 19.26 & 19.81 & 19.83 & -0.67 & G 2486; MPG 640 \\
\hline
\end{tabular}

Notes. $\uparrow$ See Fig. 3 for star positions.

References. $\ddagger$ G: stands for Gouliermis et al. (2006); MPG: refers to Massey et al. (1989).

concentrated towards this part of N66 (Staveley-Smith et al. 1997; Stanimirović et al. 1999; Gouliermis et al. 2008). The H I cloud is probably in contact with the ridge.

Figure 2 presents a high-resolution composite image of the N66A H II region extracted from the above-mentioned HST ACS archive data. The compact H II region is $\sim 10^{\prime \prime}$ in diameter, corresponding to $\sim 3 \mathrm{pc}$. It contains a strong absorption lane. Interestingly, two bright stars, labelled \#1 and \#2, are located towards the central part of the region, above the dust lane (see also Fig. 3). Separated by 0.7 ( 0.2 pc), they are the main exciting stars of the HII region, as shown in Sect. 3.3. A number of fainter stars are seen across the face of the region. These stars are quite bright on the HST ACS/HRC image in the ultraviolet obtained with $F 220 W$ and $F 330 W$ filters. Five other stars in the UV images (\#3 to \#7) should also be OB stars associated with N66A, as suggested by their photometry, presented in Table 2, which also displays the positions.

\subsection{Physical parameters}

The total $\mathrm{H} \beta$ flux of N66A was derived using the following procedure. First we calculated the relative $\mathrm{H} \beta$ flux in an imaginary $1^{\prime \prime}$ slit passing through the $\mathrm{H} \beta$ image with respect to the total flux emitted by the whole N66A region. This value was then compared with the absolute flux obtained from the spectra. In both cases, a mean flux measured for the NS and EW orientations of the slit was used. The total $\mathrm{H} \beta$ flux thus obtained was $F(\mathrm{H} \beta)=2.0 \times 10^{-12} \mathrm{erg} \mathrm{cm}^{-2} \mathrm{~s}^{-1}$. Considering the extinction law for the LMC (Howarth 1983), we computed the reddening corrected intensity $I(\mathrm{H} \beta)=3.6 \times 10^{-12} \mathrm{erg} \mathrm{cm}^{-2} \mathrm{~s}^{-1}$. We derived a luminosity of $1.6 \times 10^{36} \mathrm{erg} \mathrm{s}^{-1}$, or $400 L_{\odot}$, for N66A at $\mathrm{H}(\beta)$. This luminosity corresponds to a flux of $3.9 \times 10^{47}$ $\mathrm{H} \beta$ photons $\mathrm{s}^{-1}$, or a Lyman continuum flux of $3.4 \times 10^{48}$ photons $\mathrm{s}^{-1}$ for the star, assuming that the $\mathrm{H}$ II region is ionization bounded. The exciting star needed to provide this flux is of spectral type about O7.5 V (Martins et al. 2005) (see also Sect. 3.3).

A number of the derived physical parameters of N66A are summarized in Table 1. The mean angular radius of the H II region, corresponding to the $F W H M$ of cross-cuts through the $\mathrm{H} \alpha$ image, is given in Col. 1. The corresponding linear size, obtained using a distance modulus of $m-M=18.94 \mathrm{mag}$ (Laney \& Stobie 1994) is presented in Col. 2. The dereddened $\mathrm{H} \beta$ flux obtained from a reddening coefficient $c(\mathrm{H} \beta)=0.26$ is given in Col. 3 . The electron temperature calculated from the forbidden lines ratio [O III] $\lambda \lambda 4363 /(4959+5007)$, with an accuracy of $4 \%$, is given in Col. 4. The electron density, estimated from the ratio of the [S II] doublet $\lambda \lambda 6717 / 6731$, is presented in Col. 5. It is accurate to $\sim 80 \%$. It is well-known that the [S II] lines characterize the low-density peripheral zones of H II regions (see below for corroboration). Column 6 gives the rms electron density calculated from the $\mathrm{H} \beta$ flux, the radius, $r$, and the electron temperature, $T_{\mathrm{e}}$, assuming that the $\mathrm{H}$ II region is an ionizationbounded Strömgren sphere. For comparison reasons, an electron density of $50 \pm 30 \mathrm{~cm}^{-3}$ was found from our long-slit spectra far from N66A, towards the inner parts of the N66 giant region. This agrees well with the mean value of $60 \mathrm{~cm}^{-3}$, as estimated for the whole N66 region by Tsamis et al. (2003) using the above-mentioned ratio of the sulphur doublet, as well as a density of $80 \mathrm{~cm}^{-3}$ for N66 reported by Reid et al. (2006). Using the line ratio $[\mathrm{Cl} \mathrm{III}] \lambda \lambda 5537 / 5517$, which identifies denser zones, a density of $3700 \mathrm{~cm}^{-3}$ is found for N66 (Tsamis et al. 2003). Furthermore, the total mass of the ionized gas, calculated from the $\left\langle n_{\mathrm{e}}\right\rangle$ with the previously noted Strömgren sphere assumption is presented in Col. 7. The ionization is produced by Lyman continuum photon flux given in Col. 8 .

The most extincted part of the N66A region is the dust lane (Sect. 3.1), where the $\mathrm{H} \alpha / \mathrm{H} \beta$ ratio reaches a value of 5.0 corresponding to $A_{v}=1.7 \mathrm{mag}$. Outside the lane, the ratio is smaller in particular around the main exciting stars. The average value of the Balmer decrement towards N66A is about 3.7 $\left(A_{v}=0.8 \mathrm{mag}\right)$. We note that this ratio is higher than towards other areas of the $\mathrm{H}$ II region N66. As for the [O III] $/ \mathrm{H} \beta$ ratio, it fluctuates around 4.5 and reaches at the north-eastern border of N66A the value of 5 .

\subsection{Spectral classification}

Spectral classification was derived for five massive stars towards N66A. These stars were extracted from long-slit spectra. Four spectra belong to grating \#12 (N66A-1, MPG 455, MPG 595, 


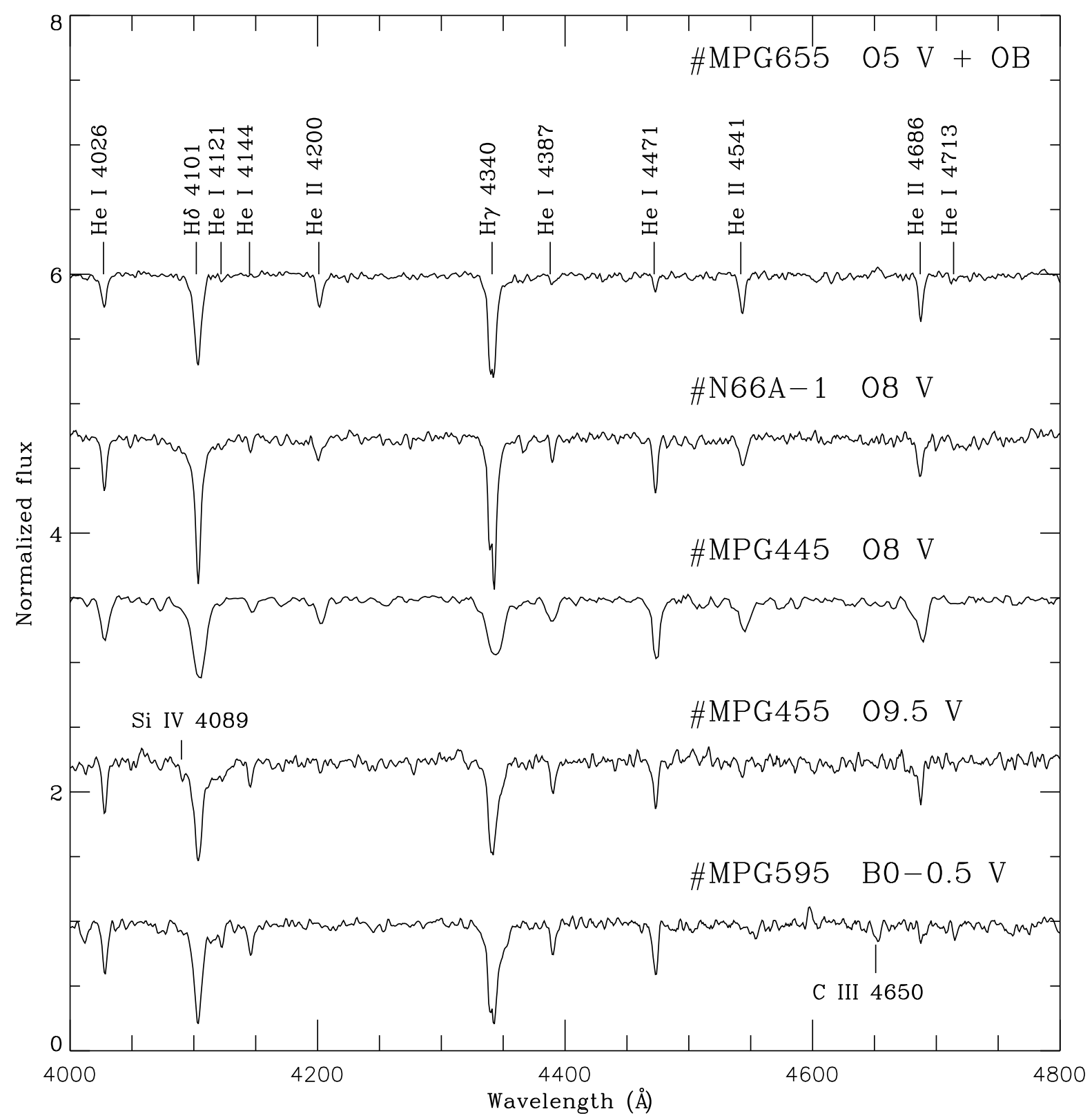

Fig. 4. Spectrograms of the classified massive stars towards SMC N66A.

MPG 655) and one spectrum (MPG 445) to grating \#4. The identification of stars along the slits were based on monitor sketches drawn during the observations. The spectral classification was performed using the criteria stated by Walborn \& Fitzpatrick (1990). The rectified spectrograms, corrected for the nebulosity background, are displayed in Fig. 4. The results are summarized in Table 3, which also gives the corresponding astrometric and photometric information as well as classifications from previous studies when available. We note that the broader lines of MPG 445 is an instrumental effect (grating \#4).

A particularly interesting star in this study, i.e. N66A-1, was classified as O8 V. This spectral type agrees well with that based on the stellar Lyman continuum derived from the $\mathrm{H} \beta$ emission of the H II region. However, the latter result is probably an underestimate because some of the ionizing photons were missed. In particular, local dust absorbs a portion of the photons and, in addition, since the H II region is not fully ionization-bounded, some of the Lyman continuum photons escape into the interstellar medium. This implies that the spectral classification based on spectroscopy is also an underestimate. This is probably because the spatial resolution of the spectra does not match that of the HST ACS images, which show that the main exciting stars are separated by 0.7 . In other words, the spectrum of N66A-1 is contaminated by N66-2. If star \#2 were of later type than star \#1, the latter would be earlier than $08 \mathrm{~V}$.

\section{Discussion}

N66A is clearly the most compact H II region of the N66 complex in the optical. Its relative compactness, brightness, and location suggest that it is probably a relatively younger generation in the N66 complex. It should belong to a distinct and rare class of $\mathrm{HII}$ regions in the Magellanic Clouds (MCs) called High-Excitation "Blobs", or HEBs 
Table 3. Stars classified towards N66A.

\begin{tabular}{llllllll}
\hline \hline Star ID & $\alpha(2000)$ & $\delta(\mathrm{J} 2000)$ & $V$ & $V-I$ & $B-V$ & Previous classification $^{\dagger}$ & This work $^{\text {Th }}$ \\
\hline N66A-1 & $00: 59: 14.93$ & $-72: 11: 01.9$ & 15.97 & -0.18 & & & O8 V \\
MPG 445 & $00: 59: 04.79$ & $-72: 11: 03.1$ & 15.33 & & -0.18 & O7.5 V (a) & O8 V \\
MPG 455 & $00: 59: 05.44$ & $-72: 10: 42.4$ & 15.25 & & -0.19 & & O9.5 V \\
MPG 595 & $00: 59: 11.91$ & $-72: 10: 55.8$ & 15.62 & & -0.20 & B0 V (b) & B0-0.5 V \\
MPG 655 & $00: 59: 15.51$ & $-72: 11: 11.6$ & 14.82 & & -0.15 & O6 V (b) & O5 V + OB \\
\hline
\end{tabular}

References. $\dagger$ (a) Niemela et al. (1986); (b) Massey et al. (1989).

(see Heydari-Malayeri et al. 2010, for a review). In contrast to the typical H II regions of the MCs, which are extended structures with sizes of several arc minutes corresponding to physical scales of more than $50 \mathrm{pc}$ and powered by a large number of exciting stars, HEBs are relatively dense and small regions of $\sim 5^{\prime \prime}$ to $10^{\prime \prime}$ in diameter in the optical, corresponding to $\sim 1.5$ to $3.0 \mathrm{pc}$ and excited by a much smaller number of massive stars. Their excitation, derived from the $[\mathrm{O}$ III] $(\lambda \lambda 4959+5007) / \mathrm{H} \beta$ ratio, is generally larger than that of ordinary MC H II regions. For a fixed metallicity, the $[\mathrm{O} \mathrm{III}] / \mathrm{H} \beta$ ratio increases with the effective temperature of the exciting star, as well as with the gas density in homogeneous photoionization models.

These compact H II regions are also heavily affected by local dust compared to other ionized features of the complex in which they are hosted (Heydari-Malayeri et al. 1988; Israel \& Koornneef 1991). This is also the case for N66A, which is marked by a prominent absorption lane of local dust crossing the whole nebula. The two other known examples of HEBs in the SMC are N88A and N81, which were also observed with HST (Heydari-Malayeri et al. 1999a,b, 2002).

HEBs are usually located adjacent to ordinary giant $\mathrm{H}$ II regions or seen lying across them. This implies that they form as a consequence of triggering by a previous generation of massive stars in the complex. Simple reasoning suggests that HEBs and their small exciting clusters are formed from the material remaining after a preceding massive-star formation event. More specifically, the apparent association of N66A with the compressed ionized front and the absorption lane, both centered on the NGC 346 cluster, suggests that N66A is a secondary, younger generation of stars. This implies that the exciting star(s) of N66A have formed according to the sequential star-formation model (Elmegreen \& Lada 1977; Whitworth et al. 1994). However, since low-mass PMS candidates have also been reported in this region, other induced star formation scenarios, such as shock waves impacting molecular cores (Vanhala \& Cameron 1998) and/or radiation-driven implosion of molecular cores (Bertoldi 1989; Kessel-Deynet \& Burkert 2003; Miao et al. 2006; Mookerjea \& Sandell 2009) are or may have been at work. Several outstanding examples of triggered massive star formation in the Galaxy were recently studied by Deharveng et al. (2009, and references therein).

As shown above (Sect. 3), a couple of exciting stars are discovered inside N66A by high-resolution HST ACS images and NTT spectroscopy. Other undetected stars may be enshrouded in the gas and dust of N66A. In this respect Simon et al. (2007) report two YSO candidates towards N66A (Fig. 3) from their analysis of the Spitzer survey. Using automatic processing, they detect a total of 1645 sources in the whole N66 region, among which some 50 embedded YSO candidates. Their automatic detection is based on spectral energy distribution (SED) fitting to 5 data points, which represent the fluxes in the Spitzer bands (at 3.6, 4.5, 5.8, 8.0, and $24 \mu \mathrm{m}$ ) with spatial resolutions from $1^{\prime \prime} 6$ to $6^{\prime \prime}$ (Sect. 2.3.2). Although the presence of YSOs is fully consistent with the star formation activity in N66A, their verification requires a detailed inspection of the Spitzer images. The Spitzer images in those bands uncover a particularly luminous object lying at the position of the compact H II region N66A. It is indeed one of the most luminous IR sources in the whole field (13000 $\left.L_{\odot}\right)$ and has a PSF of $\sim 3$ '. $6^{\prime}$ in its elongated direction. The second candidate YSO, lying at $\sim 2$ '. 4 (2 pix) from the first object, cannot be firmly detected in the Spitzer images. No concrete substructure indeed shows up in the images on this scale. In addition, the derived IRAC colors for N66A, [3.6]-[4.5] = $0.40 \mathrm{mag}$, [5.8] $-[8.0]=1.59 \mathrm{mag}$, and [3.6] $-[8.0]=3.56 \mathrm{mag}$ (Sect. 2.3.2) do not satisfy the first criterion for the YSO-class membership stated by Simon et al. (2007). From the [5.8]-[8] versus [3.6][4.5] color diagram and based on model calculations (Whitney et al. 2004), N66A also does not appear to belong definitely to the class of protostars. The IRAC colors of N66A agree perfectly with those of other HEBs, as studied by Charmandaris et al. (2008). We note that N66A is above all a very bright H II region with strong nebular emission lines affected by heavy extinction from local dust. Therefore, detecting a YSO inside the $\mathrm{H}$ II region using IRAC colors seems hazardous unless the YSO is the dominant source inside N66A, which is obviously not the case. Anyhow, the correspondence of the color of this bright H II region with those of a YSO would be a coincidence.

The suggestion by the present work of a relatively young age for the exciting star(s) of N66A disagrees with the finding of Sabbi et al. (2007) according to which all the subclusters of the N66 complex (except one) have the same age, i.e. $3 \pm 1$ Myr. Sabbi et al. (2007)'s subcluster Sc 10 overlaps N66A, in which they count a total of 61 low-mass PMS objects in an area $1.6 \mathrm{pc}$ in radius. To estimate the ages, they fit the color-magnitude diagrams of the subclusters (HST ACS images F555W and F814W) with Padua isochrones (Bertelli et al. 1994). They also use PMS isochrones (Siess et al. 2000) to evaluate the ages of the various subclusters. They note that compared to other subclusters, Sc 10 appears redder, suggesting either that it is a few million years older or that it is coeval with the others but affected by higher extinction. They maintain that the magnitudes and colors of the PMS stars in Sc 10 appear too bright and red to be compatible with a stellar population older than $\sim 4$ Myr. They therefore attribute an age of $3 \pm 1 \mathrm{Myr}$ to this subcluster, as for others, and do not consider a younger age. At the same time, they note that "Some of these associations (i.e., Sc 10 and 12) appear still embedded in dust and fuzzy nebulosities and are probably sites of recent or even still ongoing star formation." This means that they do not preclude the possibility of a younger age. Once again, one should be cautious when interpreting the color-magnitude diagrams of objects lying in the face of a very bright H II region, because the colors may be contaminated by nebular emission.

Our suggestion of a younger age for the exciting stars of N66A, however agrees perfectly with other studies of this region. In particular, Gouliermis et al. (2008) argue that the entire N66 region may host younger star formation events induced from the 
east, where the SNR B0057-724 lies (Reid et al. 2006; Nazé et al. 2002; Danforth et al. 2003; Nazé et al. 2004). There is indeed a large $\mathrm{H}$ I hole there, which is offset from the central parts of N66, and interesting enough, N66A lies on the triggered formation arc suggested to be associated with a shock wave coming from the direction of the SNR (their Fig. 1). An expanding H II region or a bubble blown by the winds of the massive progenitor of the SNR B0057-724 and possibly the W-R binary HD 5980 and the O7 Iaf+ star Sk 80 (Walborn \& Fitzpatrick 1990) may be the stimulating agent. There are therefore stars of a range of ages in the N66 complex, HD 5980 and Sk 80 being older than the NGC 346 cluster, which has not yet produced any WN stars.

\section{Concluding remarks}

We have used imaging and spectroscopy in the optical with the ESO NTT as well HST ACS and Spitzer archive data to study N66A. This compact H II region is quite a distinctive object in N66 (NGC 346), the pre-eminent starburst region of the SMC. We have presented a global view of the whole region and emphasized the importance of N66A. We derived a number of the physical characteristics of N66A, and for the first time using spectroscopy the spectral classification of the main exciting star of N66A. It is a dwarf massive star of type earlier than O8.

We have argued that N66A is probably produced by a recent massive star formation in N66. Its exciting stars are most likely to have been triggered by the action of shocks caused by a previous generation of massive stars. Moreover, N66A belongs to a rare class of compact $\mathrm{H}$ II regions in the MCs, called HEBs (High-Excitation Blobs). Only two other HEBs have so far been detected in the MCs.

Acknowledgements. We wish to thank Prof. Vassilis Charmandaris, Dept. of Physics, Univ. of Crete, Greece, for discussions and help in the exploitation of Spitzer data. We are also grateful to Dr. Nolan R. Walborn, Space Telescope Institute, Baltimore, USA, for advices about the spectral classification of the massive stars. We are indebted to Dr. Frédéric Meynadier, GEPI, Paris Observatory, France, for a preliminary reduction of the NTT images. And finally we would like to thank an anonymous referee whose helpful comments considerably improved the final version.

\section{References}

Bertelli, G., Bressan, A., Chiosi, C., et al. 1994, A\&AS, 106, 275 Bertoldi, F. 1989, ApJ, 346, 735

Bolatto, A. D., Simon, J. D., Stanimirović, S., et al. 2007, ApJ, 655, 212

Bouret, J.-C., Lanz, T., Hillier, D. J., et al. 2003, ApJ, 595, 1182

Charmandaris, V., Heydari-Malayeri, M., \& Chatzopoulos, E. 2008, A\&A, 396, 255

Contursi, A., Lequeux, J., Cesarsky, D., et al. 2000, A\&A, 362, 310

Crowther, P. A., \& Bibby, J. L. 2009, A\&A, 499, 455

de Boer, K. S., \& Savage, B. D. 1980, ApJ, 238, 86

Danforth, C. W., Sankrit, R., Blair, W. P., Howk, J. C., \& Chu, Y.-H. 2003, ApJ, 586, 1179
Deharveng, L., Zavagno, A., Schuller, F., et al. 2009, A\&A, 496, 177 Elmegreen, B. G., \& Lada, C. J. 1977, ApJ, 214, 725

Evans, C. J., Lennon, D. J., Smartt, S. J., \& Trundle, C. 2006, A\&A, 456, 623 Gouliermis, D. A., Dolphin, A. E., Brandner, W., \& Henning, Th. 2006, ApJS, 166,549

Gouliermis, D. A., Chu, Y.-H., Henning, T., et al. 2008, ApJ, 688, 1050

Gouliermis, D. A., Bestenlehner, J. M., Brandner, W., \& Henning 2010, A\&A, 515, A56

Henize, K. G. 1956, ApJS, 2, 315

Hennekemper, E., Gouliermis, D. A., Henning, T., et al. 2008, ApJ, 672, 914

Heydari-Malayeri, M., \& Hutsemékers, D. 1991, A\&A, 243, 401

Heydari-Malayeri, M., Le Bertre, T., \& Magain, P. 1988, A\&A, 195, 230

Heydari-Malayeri, M., Melnick, J., \& Martin, J.-M. 1990, A\&A, 234, 99

Heydari-Malayeri, M., Rosa, M. R., Zinnecker, H., Deharveng, L., \& Charmandaris, V. 1999, A\&A, 344, 848

Heydari-Malayeri, M., Charmandaris, V., Deharveng, L., Rosa, M. R., \& Zinnecker, H. 1999, A\&A, 347, 841

Heydari-Malayeri, M., Rosa, M. R., Schaerer, D., Martins, F., \& Charmandaris, V. 2002, A\&A, 381, 951

Heydari-Malayeri, M., Rosa, M. R., Charmandaris, V., et al. 2010, The Impact of HST on European Astronomy, ed. F. D. Macchetto, Astrophys. Space Sci. Proceed., 31

Howarth, I. D. 1983, MNRAS, 203, 301

Israel, F. P., \& Koornneef, J. 1991, A\&A, 248, 404

Kennicutt, R. C. 1984, ApJ, 287, 116

Kessel-Deynet, O., \& Burkert, A. 2003, MNRAS, 338, 545

Laney, C. D., \& Stobie, R. S. 1994, MNRAS, 266, 441

Le Coarer, E., Rosado, M., Georgelin, Y. P., et al. 1993, A\&A, 280, 365

Martins, F., Schaerer, D., \& Hillier, D. J. 2005, A\&A, 436, 1049

Massey, P., Parker, J. W., \& Garmany, C. D. 1989, AJ, 98, 1305

Miao, J., White, G. J., Nelson, R., et al. 2006, MNRAS, 369, 143

Mookerjea, B., \& Sandell, G. 2009, ApJ, 706, 896

Nazé, Y., Hartwell, J. M., Stevens, I. R., et al. 2002, ApJ, 580, 225

Nazé, Y., Manfroid, J., Stevens, I. R., et al. 2004, ApJ, 608, 208

Niemela, V. S., Marraco, H. G., \& Cabanne, M. L. 1986, PASP, 98, 1133

Nota, A., Sirianni, M., Sabbi, E., et al. 2006, ApJ, 640, L29

Reid, W. A., Payne, J. L., Filipovic, M. D., et al. 2006, MNRAS, 367, 1379

Rubio, M., Lequeux, J., Boulanger, F., et al. 1996, A\&AS, 118, 263

Rubio, M., Contursi, A., Lequeux, J., et al. 2000, A\&A, 359, 1139

Sabbi, E., Sirianni, M., Nota, A., et al. 2007, AJ, 133, 44 (SSN07)

Sabbi, E., Sirianni, M., Nota, A., et al. 2007, AJ, 133, 2430 (erratum)

Schmeja, S., Gouliermis, D. A., \& Klessen, R. S. 2009, ApJ, 694, 367

Siess, L., Dufour, E., \& Forestini, M. 2000, A\&A, 358, 593

Simon, J. D., Bolatto, D., Whitney, B. A., et al. 2007, ApJ, 669, 327

Smith, C., Leiton, R., \& Pizarro, S. 2000, in, Stars, Gas, and Dust in Galaxies: Exploring the Links, ed. D. Alloin, et al. (San Francisco: ASP), ASP Conf. Ser., 221,83

Stanimirović, S., Staveley-Smith, L., Dickey, J. M., et al. 1999, MNRAS, 302, 417

Staveley-Smith, L., Sault, R. J., Hatzidimitriou, et al. 1997, MNRAS, 289, 225

Tsamis, Y. G., Barlow, M. J., Liu, X.-W., Danziger, I. J., \& Storey, P. J. 2003, MNRAS, 338, 687

Vanhala, H. A. T., \& Cameron, A. G. W. 1998, ApJ, 508, 291

Walborn, N. R., \& Blades, J. C. 1986, ApJ, 304, L17

Walborn, N. R., \& Fitzpatrick, E. L. 1990, PASP, 102, 379

Walborn, N. R., Lennon, D. J., Heap, S. R., et al. 2000, PASP, 112, 1243

Walborn, N. R., Howarth, I. D., Lennon, D. J., et al. 2002a, AJ, 123, 2754

Walborn, N. R., Fullerton, A. W., Crowther, P. A., et al. 2002b, ApJS, 141, 443

Whitney, B., Indebetouw, R., Bjorkman, J. E., \& Wood, K. 2004, ApJ, 617, 1177

Whitworth, A. P., Bhattal, A. S., Chapman, S. J., et al. 1994, MNRAS, 268, 291

Ye, T., Turtle, A. J., Kennicutt, R. C., Jr. 1991, MNRAS, 249, 722 